\title{
Composition and antimicrobial activity of the essential oil and water extract from Japanese wild Rosa rugosa
}

\author{
Masahiko Nagaki*, Yoshifumi Goto, Takashi Narita, \\ Jun Kawakami, and Ryo Miyamoto \\ Graduate School of Science and Technology, Hirosaki University, \\ 3 Bunkyo-cho, Hirosaki, Aomori 036-8561, Japan \\ *Fax: 81-172-39-3947, e-mail: nagaki@cc.hirosaki-u.ac.jp
}

\begin{abstract}
The essential oils and distilled-water extracts of Rosa rugosa flower, leaf, and fruit were analyzed by flame ionization detector-gas chromatography and mass spectrometer-gas chromatography. The principle components in the flower oil and water were citronellyl acetate, citronellol, and geranyl acetate, and citronellol and geraniol, respectively. For the leaf oil and water, it was $\gamma$-muurolene and geraniol and linalool, respectively. The main component in fruit water was terpinen-4-ol. Fruit oil, on the other hand, could not be extracted. Additionally, combining the Rosa rugosa distilled-water with one of the isoprenoids geraniol, farnesol, or citral showed higher antibacterial activity than the isoprenoid alone.

Key words: Rose rugosa; hydrodistillation; essential oil; distillated water; antimicrobial activity;
\end{abstract}

\section{INTRODUCTION}

Roses have been a popular aesthetic since ancient times. Even today, their essential oils are regularly used for cosmetics, the most frequent being that from the extract of Rosa damascene [1], which is composed mostly of citronellol and geraniol [2].

R. rugosa, a native of northeastern Asia that includes the popular garden rose Pink Grootendorst, is also a popular species for this purpose [3, 4]. Because of its potential cosmetic and even pharmaceutical applications, the chemical components, composition, and phytochemistry of $R$. rugosa native to certain countries have been determined[5-9]. However, $R$. rugosa native to Japan is an exception. We therefore report here the chemical components of the essential oils and distilled water extracts from wild Japanese R. rugosa. Additonally, seeing that a number of studies have reported the antimicrobial activity of essential oil ingredients from a wide range of plants[10-16] and that we recently reported the antibacterial and antifungal activities of several isoprenoids [17], we investigated the antimicrobial activity of these components by conducting assays on the rugosa rose essential oil and distilled-water when combined with specific isoprenoids to identify potential synergistic effects, which may indicate cosmetic and/or pharmaceutical benefits.

\section{EXPERIMENTAL}

\subsection{Plant materials}

Wild $R$. rugosa flowers were collected in Ajigasawa, Aomori, Japan, on June, 2009. The flowers were hydrodistilled for 6 hours, resulting in $0.145 \mathrm{~g}(0.0058 \%$ fresh distillate weight $)$ and $0.64 \mathrm{~g}(0.04 \%)$ of essential oils yielded from 2.5 $\mathrm{kg}$ of flower and $1.6 \mathrm{~kg}$ of leaf, respectively.

\subsection{Extraction of distilled-water}

Distilled water extracts were extracted 4 times with hexane $(300 \mathrm{ml}$ hexane/L distilled water $)$.

\subsection{Analysis}

Essential oils and distillated water extracts were analyzed by flame ionization detector-gas chromatography (GC-FID). The GC-FID analysis was performed using a Hitachi Gas Chromatograph G-5000A (Hitachi, Tokyo, Japan). A $60 \mathrm{ml} / \mathrm{min}$ nitrogen carrier flow rate was passed through a poly(alkylene glycol) column $(30 \mathrm{~m} \times$ $0.25 \mathrm{~mm}$ i.d.) using a $10{ }^{\circ} \mathrm{C} / \mathrm{min}$ gradient that began with a $5 \mathrm{~min}$ hold at $40{ }^{\circ} \mathrm{C}$ and ended upon reaching $200{ }^{\circ} \mathrm{C}$. Injector and detector temperatures were $150{ }^{\circ} \mathrm{C}$ and $250{ }^{\circ} \mathrm{C}$, respectively. Mass spectrometer-gas chromatography (GC-MS) analysis was conducted on a JEOL Q1000GC-Mk II Mass Spectrometer, (Japan Electron Optics Laboratory Co. Ltd., Japan) that included an HP-5 column $(30 \mathrm{~m} \times \phi$ $0.32 \mathrm{~mm}$ ) using a $1 \mathrm{ml} / \mathrm{min}$ helium carrier flow rate. The temperature was programmed to deliver a $15{ }^{\circ} \mathrm{C} / \mathrm{min}$ gradient that began with a $4.7 \mathrm{~min}$ hold at $50{ }^{\circ} \mathrm{C}$ and ended with a 2 min hold at $280{ }^{\circ} \mathrm{C}$. Injector and GC-transfer line temperatures were both set to $200{ }^{\circ} \mathrm{C}$.

Separated components were identified by comparing retention times and mass spectra with laboratory data and an NIST library (http://www.nist.gov/srd/nist1a.cfm). Accordance of the retention times in the GC-MS was 
confirmed by co-injecting the reagent with an n-alkane $\left(\mathrm{C}_{7}-\mathrm{C}_{40}\right)$ retention index. The relative amounts of the components were calculated from their respective GC-MS peak areas.

\subsection{Assay of antimicrobial activity}

Antimicrobial assays were performed in accordance with the agar plate dilution method as recommended by the Japanese Society of Chemotherapy[18-20]. Bacteria were inoculated into $10 \mathrm{ml}$ of Müeller-Hinton Broth medium (MHB, Difco ${ }^{\mathrm{TM}}$, Becton Dickinson (BD), USA) and cultured at $37^{\circ} \mathrm{C}$ for $24 \mathrm{~h}$ without shaking. Each isoprenoid was dissolved in DMSO or a $5 \%$ aqueous solution of the surfactant HCO-50 (w/v) (Nikko Chemicals Company Ltd., Japan). Then, $120 \mu \mathrm{L}$ of the dissolved isoprenoid solution was added to $12 \mathrm{ml}$ Müeller-Hinton Agar (MHA) culture medium. For combination assays, water obtained from the flower of rugosa rose by hydrodistillation was used instead of regular distilled water for the MHA culture medium. The optical density (OD) of bacterial cultures at 550 $\mathrm{nm}$ was determined using a Smart $\mathrm{Spec}^{\mathrm{TM}} 3000$ (Bio-Rad Laboratories, USA) prior to performing colony counts. Cultures were adjusted to $1 \times 10^{6}$ colony-forming units $(\mathrm{CFU}) / \mathrm{ml}$, and colonies were counted after incubation at $37{ }^{\circ} \mathrm{C}$ for $20 \mathrm{~h}$. Fungi were cultured in Sabouraud Dextrose agar medium (Eiken Chemical Co., Ltd., Japan) at $25-27^{\circ} \mathrm{C}$.

The Minimal Inhibitory Concentration (MIC) at which a molecule exerted antimicrobial activity was defined as less than $800 \mu \mathrm{g} / \mathrm{mL}$, in accordance with the criterion set forth by the Japanese Society of Chemotherapy [20-22]. Media were sterilized using an STH 364 FA autoclave (Advantec Toyo Kaisha, Ltd.).

\subsection{Bacterial strains}

Gram-positive bacteria used were as follows: Staphylococcus aureus 209P, and S. aureus 834 (methicillin resistant - MRSA) Gram-negative strains were E. coli IFO-3806, and Shigella sonnei.

\section{RESULTS AND DISCUSSION}

Table 1 gives a summary of the components from essential oil and distillated water extracts from $R$. rugosa flower. We identified 27 components in the essential oil, the main being citronellyl acetate $(12.1 \%)$, citronellol $(7.7 \%)$ and geranyl acetate $(5.5 \%)$. In the distilled water, 22 components were identified, with the main being citronellol (31.4\%). Other sweet monoterpene alcohols like geraniol, nerol and linalool, had higher percentages in the floral water extract than in the essential oil. In contrast, acetates such as citronellyl acetate and geranyl acetate were proportionally less in the flower water extract than in the essential oil. Finally, the flower water extract saw a significant increase in the proportion of 2-phenylethanol, a popular ingredient used in fragrances. The increased alcohol percentages may imply pharmacological benefits, as we recently reported several
Table 1. Components of essential oil and distilled water extract from $R$. rugosa flower

\begin{tabular}{|c|c|c|c|c|c|c|}
\hline No. & Components & EO (\%) & DW ex. (\%) & RT & RI & $\mathrm{CI}$ \\
\hline 1 & $\beta$-myrcene & - & 0.8 & $6: 56$ & 994 & 0 \\
\hline 2 & $p$-cymene & 0.7 & 1.4 & $7: 29$ & 1027 & O \\
\hline 3 & limonene & 1.6 & 3.7 & $7: 34$ & 1032 & 0 \\
\hline 4 & $\gamma$-terpinene & - & 0.4 & 8:03 & 1063 & - \\
\hline 5 & acetophenone & - & 0.6 & $8: 10$ & 1071 & - \\
\hline 6 & cis-linalool oxide & - & 0.5 & $8: 17$ & 1078 & - \\
\hline 7 & linalool & 0.5 & 9.0 & $8: 38$ & 1100 & O \\
\hline 8 & 2-phenylethanol & 0.9 & 8.1 & $8: 50$ & 1116 & O \\
\hline 9 & menthone & 0.6 & 0.5 & $9: 22$ & 1158 & 0 \\
\hline 10 & terpinen-4-ol & - & 0.7 & $9: 40$ & 1183 & 0 \\
\hline 11 & $\alpha$-terpineol & - & 5.2 & $9: 49$ & 1195 & 0 \\
\hline 12 & citronellol & 7.7 & 31.4 & 1011 & 7 & 0 \\
\hline 13 & nerol & 1.4 & 11.1 & 10.15 & 1221 & $\mathrm{O}$ \\
\hline 14 & geraniol & 1.5 & 13.4 & $10: 30$ & 1254 & O \\
\hline 15 & 2-phenyl ethyl acetate & - & 0.7 & $10: 33$ & 1260 & - \\
\hline 16 & citronellyl formate & 1.4 & - & $10: 42$ & 1270 & - \\
\hline 17 & citronellyl acetate & 12.1 & 0.8 & $11: 29$ & 1355 & 0 \\
\hline 18 & eugenol & - & 4.1 & $11: 34$ & 1365 & $\mathrm{O}$ \\
\hline 19 & geranyl acetate & 5.5 & 0.2 & $11: 46$ & 1385 & 0 \\
\hline 20 & methyl eugenol & 0.5 & 2.3 & $11: 58$ & 1405 & - \\
\hline 21 & $\beta$-cedrene & 0.9 & - & $12: 08$ & 1426 & - \\
\hline 22 & $\alpha$-bergamotene & 1.6 & - & $12: 17$ & 1443 & - \\
\hline 23 & epi- $\beta$-santalene & 0.6 & - & $12: 23$ & 1454 & - \\
\hline 24 & $\gamma$-muurolene & 6.1 & - & $12: 31$ & 1469 & - \\
\hline 25 & $\alpha$-curcumene & 1.0 & - & $12: 41$ & 1489 & - \\
\hline 26 & 2-tridecanone & 6.0 & - & $12: 45$ & 1496 & - \\
\hline 27 & $\begin{array}{l}\text { 8-isopropyl-1,5-dimethyl } \\
\text { cyclodeca-1,5-diene }\end{array}$ & 5.5 & - & $12: 53$ & 1520 & - \\
\hline 28 & nerolidol & 0.7 & - & $12: 59$ & 1524 & - \\
\hline 29 & caryophyllene oxide & 0.3 & - & $13: 32$ & 1590 & - \\
\hline 30 & widdrol & - & 0.4 & $13: 46$ & 1620 & - \\
\hline 31 & nerolidyl acetate & 0.5 & - & $14: 10$ & 1672 & - \\
\hline 32 & $\beta$-bisabolol & 0.5 & 0.1 & $14: 21$ & 1696 & - \\
\hline 33 & 2-pentadecanone & 1.4 & - & $14: 23$ & 1700 & - \\
\hline 34 & farnesol & 1.0 & 0.2 & $14: 35$ & 1728 & 0 \\
\hline 35 & farnesal & 0.4 & - & $14: 44$ & 1748 & - \\
\hline 36 & hexadecanol acetate & 0.4 & - & $16: 33$ & 2008 & - \\
\hline- & n-alkane $\left(\mathrm{C}_{21} \sim \mathrm{C}_{27}\right)$ & 23.2 & - & - & - & - \\
\hline \multirow[t]{2}{*}{ - } & components less than $0.3 \%$ & 0.6 & 0.6 & - & - & - \\
\hline & Total & 85.2 & 96.3 & & & \\
\hline
\end{tabular}

Components less than $0.3 \%$ : $\alpha$-pinene, $\alpha$-terpinene, trans-rose oxide, neral, geranial, hexadecanal

For tables 1-3:EO, yield (\%) of essential oil DWex, yeild (\%) of distilled-water extract $\mathrm{RT}$, retention time

$\mathrm{RI}$, retention index

CI: co-injection with authentic samples 
Table 2. Components of essential oil and distilled water extract from $R$. rugosa leaf.

\begin{tabular}{|c|c|c|c|c|c|c|}
\hline No. & Components & EO (\%) & DW ex. $(\%)$ & RT & RI & $\mathrm{CI}$ \\
\hline 1 & trans-linalool oxide & - & 0.7 & $8: 16$ & 1077 & - \\
\hline 2 & cis-linalool oxide & - & 0.5 & $8: 30$ & 1087 & - \\
\hline 3 & linalool & 0.2 & 3.9 & $8: 39$ & 1101 & $\mathrm{O}$ \\
\hline 4 & nonanal & 0.3 & 0.3 & $8: 43$ & 1107 & - \\
\hline 5 & terpinen-4-ol & $\operatorname{tr}$ & 1.2 & $9: 39$ & 1181 & $\mathrm{O}$ \\
\hline 6 & 2-methyl acetophenone & $\operatorname{tr}$ & 0.3 & $9: 44$ & 1188 & - \\
\hline 7 & $\alpha$-terpineol & $\operatorname{tr}$ & 1.5 & 9:49 & 1195 & 0 \\
\hline 8 & citronellol & $\operatorname{tr}$ & 0.4 & $10: 13$ & 1231 & 0 \\
\hline 9 & 2-butanoic acid, hexyl ester & 0.1 & - & $10: 27$ & 1253 & - \\
\hline 10 & geraniol & 0.4 & 4.2 & $10: 29$ & 1256 & 0 \\
\hline 11 & piperitone & - & 0.8 & $10: 32$ & 1260 & - \\
\hline 12 & geranial & - & 0.5 & $10: 41$ & 1275 & $\mathrm{O}$ \\
\hline 13 & geranyl formate & $\operatorname{tr}$ & 0.5 & $10: 59$ & 1303 & - \\
\hline 14 & 4-hydroxy-3-methyl acetophenone & - & 1.3 & 11:05 & 1313 & - \\
\hline 15 & $\begin{array}{l}\text { 4-methylene-2,8,8-trimethyl-2- } \\
\text { vinyl-bicyclo[5.2.0]nonane }\end{array}$ & 1.0 & - & 11:44 & 1382 & - \\
\hline 16 & $\beta$-cedrene & 1.7 & - & $12: 08$ & 1426 & - \\
\hline 17 & $\alpha$-cedrene & 0.7 & - & $12: 12$ & 1434 & - \\
\hline 18 & bergamotene & 1.2 & - & $12: 16$ & 1442 & - \\
\hline 19 & elemene & 0.9 & - & $12: 19$ & 1447 & - \\
\hline 20 & $\alpha$-amorphene & 3.7 & - & $12: 23$ & 1455 & - \\
\hline 21 & $\gamma$-muurolene & 10.4 & - & $12: 31$ & 1470 & - \\
\hline 22 & $\alpha$-curcumene & 1.2 & - & $12: 41$ & 1489 & - \\
\hline 23 & spathulenol & 0.3 & - & 13:24 & 1574 & - \\
\hline 24 & caryophyllene oxide & 0.8 & - & $13: 32$ & 1590 & - \\
\hline 25 & aromadendrene oxide & 2.7 & 3.5 & 14:06 & 1663 & - \\
\hline 26 & $\alpha$-bisabolol oxide & - & 1.0 & 14:09 & 1670 & - \\
\hline 27 & Isolongifolol, methyl ether & - & 0.8 & 14:11 & 1672 & - \\
\hline 28 & $\beta$-eudesmol & 0.3 & - & 14:15 & 1683 & - \\
\hline 29 & $\alpha$-bisabolol & 1.6 & - & $14: 20$ & 1685 & - \\
\hline 30 & $\begin{array}{l}\text { 2,2,7,7-tetramethyl tricycle } \\
{[6.2 .1 .0(1,6)] \text { undec-4-en-3-one }}\end{array}$ & 3.1 & 0.9 & $14: 27$ & 1709 & - \\
\hline 31 & $\begin{array}{l}\text { eudesma-5,11(13) } \\
\text {-dien-8,12-olide }\end{array}$ & 1.4 & - & 15:00 & 1784 & - \\
\hline 32 & isophytol & 0.3 & - & 16:09 & 1948 & - \\
\hline 33 & parthenium & 0.8 & - & $16: 11$ & 1960 & - \\
\hline 34 & phytol & 1.9 & - & $17: 13$ & 2114 & - \\
\hline- & n-alkane $\left(\mathrm{C}_{23} \sim \mathrm{C}_{27}\right)$ & 1.5 & - & - & - & - \\
\hline- & components less than $0.3 \%$ & 0.9 & 1.5 & - & - & - \\
\hline & Total & 37.2 & 23.6 & & & \\
\hline
\end{tabular}

Components less than $0.3 \%$ : 1-octen-3-ol, $\beta$-myrcene, p-cymene, limonene, benzyl alcohol, trans-ocimene, benzenacetaldehyde, ocimene (isomer), 2-phenylethanol, methoxy citronellol, 2-nonenal, menthone, nonyl alcohol, decanal, neral, 2-butanoic acid, hexyl ether, nonanal dimethyl acetal, undecanal, 1,2-dihudro-1,1,6-trimethy naphthalene, eugenol, $\beta$-damascenone, 2,5-diisopropyl phenol, calamenene
Table 3. Components of essential oil and distilled water extract from $R$. rugosa fruit.

\begin{tabular}{|c|c|c|c|c|c|}
\hline No. & Components & DW ex. (\%) & RT & RI & $\mathrm{CI}$ \\
\hline 1 & $\beta$-myrcene & 1.5 & $6: 52$ & 992 & O \\
\hline 2 & limonene & 0.5 & $7: 31$ & 1029 & 0 \\
\hline 3 & trans-ocimene & 0.4 & 7:42 & 1041 & - \\
\hline 4 & ocimene & 0.6 & 7:52 & 1052 & - \\
\hline 5 & trans-linalool oxide & 0.4 & 8:14 & 1075 & - \\
\hline 6 & cis-linalool oxide & 0.4 & $8: 28$ & 1089 & - \\
\hline 7 & linalool & 15.6 & $8: 37$ & 1099 & 0 \\
\hline 8 & nonanal & 0.8 & 8:41 & 1104 & - \\
\hline 9 & 1-octen-3-yl-acetate & 0.8 & $8: 48$ & 1113 & - \\
\hline 10 & borneol & 1.0 & $9: 30$ & 1169 & $\mathrm{O}$ \\
\hline 11 & terpinen-4-ol & 19.1 & $9: 37$ & 1179 & 0 \\
\hline 12 & $\alpha$-terpineol & 6.4 & 9:47 & 1192 & 0 \\
\hline 13 & citronellol & 0.4 & $10 \cdot 1$ & 1230 & $\mathrm{O}$ \\
\hline 14 & nerol & 0.9 & 10.12 & 1200 & O \\
\hline 15 & geraniol & 2.9 & $10 \cdot 29$ & 1556 & O \\
\hline 16 & linalyl acetate & 3.4 & & & O \\
\hline 17 & lavandulyl acetate & 6.6 & $10: 51$ & 1290 & - \\
\hline 18 & carvacrol & 0.4 & $10: 58$ & 1302 & - \\
\hline 19 & neryl acetate & 1.7 & $11: 34$ & 1365 & 0 \\
\hline 20 & geranyl acetate & 3.2 & $11: 45$ & 1384 & $\mathrm{O}$ \\
\hline 21 & $\beta$-caryophyllene & 5.1 & 12:10 & 1430 & $\mathrm{O}$ \\
\hline 22 & $\alpha$-bergamotene & 0.3 & $12: 16$ & 1442 & - \\
\hline 23 & $\beta$-farnesene & 4.1 & $12: 25$ & 1458 & - \\
\hline 24 & $\gamma$-cadinene & 1.7 & $12: 59$ & 1524 & - \\
\hline 25 & calamenene & 0.3 & 13:03 & 1532 & - \\
\hline 26 & caryophyllene oxide I & 0.3 & 13:20 & 1566 & - \\
\hline 27 & caryophyllene oxide II & 3.5 & 13:36 & 1598 & - \\
\hline 28 & cubenol & 0.3 & 13:50 & 1628 & - \\
\hline 29 & $\tau$-cadinol & 4.2 & 14:01 & 1652 & - \\
\hline 30 & cis-Z- $\alpha$-bisabolene epoxide & 0.7 & 14:10 & 1672 & - \\
\hline 31 & longipinocarvone & 0.4 & $14: 49$ & 1759 & - \\
\hline 32 & $\begin{array}{l}\text { 6,10,14-trimethyl-2- } \\
\text { pentadecanone }\end{array}$ & 0.8 & $15: 27$ & 1847 & - \\
\hline \multirow[t]{3}{*}{-} & n-alkane $\left(\mathrm{C}_{21}, \mathrm{C}_{23}, \mathrm{C}_{25}, \mathrm{C}_{27}\right)$ & 1.4 & - & - & - \\
\hline & components less than $0.3 \%$ & 1.9 & - & - & - \\
\hline & Total & 92.3 & & & \\
\hline
\end{tabular}

Components less than $0.3 \%$ : ethyl tiglate, $p$-cymene, $\gamma$-terpinene, camphor, nonyl alcohol, $p$-cymen-8-ol, carvenone, geranial, undecanal, $\alpha$-caryophyllene, $\gamma$-muurolene, elemophilene, $\beta$-bisabolene, $\gamma$-eudesmol, cadalene 
monoterpene alcohols such as geraniol have antimicrobial activity even at low concentrations[17].

Furthermore, geraniol, linalool and citronellol have a tendency to emit more sweet fragrance than their acetate compounds, suggesting the flower water may be more useful for cosmetic applications.

For the essential oil of leaf (Table 2), we identified 22 components, the main being $\gamma$-muurolene $(10.4 \%)$. The leaf oil also contained geraniol $(0.4 \%)$, but at a proportion significantly less than the amount in flower oil $(1.5 \%)$. Rose like fregrance conpounds such as geraniol and citronellol were lower than flower's, and it emitted grassy smell. We found 17 components in the water extract, the most prominent being geraniol (4.2\%) and linalool (3.9\%).

Furthermore, similar to $R$. rugosa flower, the proportional amounts of geraniol and linalool in the leaf water extract were greater than that in the essential oil.

The essential oil from $R$. rugosa fruit could not be extracted. Table 3 shows the 32 components identified from the fruit water extract, the main being terpinen-4-ol (19.1\%) and linalool (15.6\%). Additionally, the fruit water was found to contain several hydrophobic alkenes such as $\beta$-caryophyllene $(5.1 \%)$ and $\beta$-farnesene $(4.1 \%)$.

Upon identifying the main components of $R$. rugosa, we next considered potential pharmacological effects. On its own, we found the distilled-water from $R$. rugosa flower showed no antimicrobial activity against either Gram positive or Gram negative bacteria.

Table 4. Geraniol antimicrobial activity against bacteria when distilled-water from $R$. rugosa flower was used in the MHA culture medium.

\begin{tabular}{ccccc}
\hline geraniol $(\mu \mathrm{g} / \mathrm{mI})$ & \multicolumn{2}{c}{$\begin{array}{c}\text { Gram-positive } \\
\text { bacteria }\end{array}$} & \multicolumn{2}{c}{$\begin{array}{c}\text { Gram-negative } \\
\text { bacteria }\end{array}$} \\
\cline { 2 - 5 } $\begin{array}{c}\text { (+distilled water } \\
\text { of } \text { R. rugosa) }\end{array}$ & S. aureus & MRSA & E. coli & S. sonnei \\
\hline $\mathbf{4 6 7}$ & + & + & + & + \\
$\mathbf{4 4 5}$ & + & + & + & + \\
$\mathbf{4 2 3}$ & + & + & + & + \\
$\mathbf{4 0 0}$ & - & - & - & - \\
$\mathbf{3 7 8}$ & - & - & - & - \\
\hline
\end{tabular}

Table 5. Antimicrobial activity of farnesol when combined with distilled-water from $R$. rugosa flower for the MHA culture medium

\begin{tabular}{ccccc}
\hline farnesol $(\mu \mathrm{g} / \mathrm{ml})$ & \multicolumn{2}{c}{$\begin{array}{c}\text { Gram-positive } \\
\text { bacteria }\end{array}$} & \multicolumn{2}{c}{$\begin{array}{c}\text { Gram-negative } \\
\text { bacteria }\end{array}$} \\
\cline { 2 - 5 } $\begin{array}{c}\text { (+distilled water } \\
\text { of } \boldsymbol{R} \text {. rugosa })\end{array}$ & S. aureus & MRSA & E. coli & S. sonnei \\
\hline 155 & + & + & - & - \\
133 & + & + & - & - \\
111 & + & + & - & - \\
89 & - & - & - & - \\
67 & - & - & - & - \\
\hline
\end{tabular}

Table 6. Antimicrobial activity of citral when combined with distilled-water from $R$. rugosa flower for the MHA culture medium

\begin{tabular}{ccccc}
\hline citral $(\mu \mathrm{g} / \mathrm{ml})$ & \multicolumn{2}{c}{$\begin{array}{c}\text { Gram-positive } \\
\text { bacteria }\end{array}$} & \multicolumn{2}{c}{$\begin{array}{c}\text { Gram-negative } \\
\text { bacteria }\end{array}$} \\
\cline { 2 - 5 } $\begin{array}{c}\text { (+distilled water } \\
\text { of } R \text {. rugosa) }\end{array}$ & S. aureus & MRSA & E. coli & S. sonnei \\
\hline 489 & + & + & + & - \\
444 & + & + & + & - \\
400 & + & + & + & - \\
356 & + & + & + & - \\
289 & + & + & + & - \\
267 & - & - & - & - \\
245 & - & - & - & - \\
222 & - & - & - & - \\
\hline
\end{tabular}

However, we have previously reported the antimicrobial activity of isoprenoids[17].

Therefore, to identify any synergistic effects, we conducted antibacterial assays by combining the distilled-water of $R$. rugosa flower and each of the three isoprenoids. This caused the MIC of geraniol in response to $S$. aureus and MRSA to decrease from $534 \mu \mathrm{g} / \mathrm{ml}$ to $423 \mu \mathrm{g} / \mathrm{ml}$ and in response to $E$. coli or $S$. sonnei to decrease from $445 \mu \mathrm{g} / \mathrm{ml}$ to $423 \mu \mathrm{g} / \mathrm{ml}$, indicating a stronger drug effect (Table 4). Similarly, the MIC for farnesol decreased from $184 \mu \mathrm{g} / \mathrm{ml}$ to $111 \mu \mathrm{g} / \mathrm{ml}$ against $S$. aureus or MRSA (Table 5). However, no change in the MIC was seen for farnesol against Gram negative bacteria when mixed with $R$. rugosa flower distilled water. This was also true for citral, where the MIC improved from 356 $\mu \mathrm{g} / \mathrm{ml}$ to $289 \mu \mathrm{g} / \mathrm{ml}$ against Gram positive bacteria, but did not change against Gram negative bacteria like E. coli. However, no change in the MIC was seen for farnesol against Gram negative bacteria when mixed with $R$. rugosa flower distilled water. This was also true for citral, where the MIC improved from 356 $\mu \mathrm{g} / \mathrm{ml}$ to $289 \mu \mathrm{g} / \mathrm{ml}$ against Gram positive bacteria, but did not change against Gram negative bacteria like E. coli (Table 6).

Therefore, we show that isoprenoids have a more potent drug effect when mixed with the flower water of R. rugosa. This is consistent with previous reports that have shown the oil of other plants produce a synergistic effect too[23-26].

\section{CONCLUSIONS}

We analyzed the components of $R$. rugosa, finding the principal components of the flower essential oil to be citronellyl acetate, citronellol, and geranyl acetate, while the principal components of the flower distilled water were citronellol and geraniol. The principal component of leaf essential oil was $\gamma$-muurolene while that of the leaf water was geraniol and linalool. While the essential oil of fruit could not be extracted, the principal component of fruit water was found to be terpinen-4-ol. More importantly, we found 
synergistic effects when combining isoprenoids such as geraniol, farnesol, and citral with the distilled water of $R$. rugosa flower, suggesting potential drug applications.

\section{ACKNOWLEDGEMENTS}

This work was supported in part by a grant from the Cosmetology Research Foundation (M.N.) and the municipal government of Ajigasawa (M. N.).

\section{REFERENCES}

[ 1] N. G. Baydar, H. Baydar, T. Debener, J. Biotechnol.,111, 263-267 (2004).

[2] K. G. D. Babu, B. Singh, V. P. Joshi, V. Singh, Flavour Fragr. J., 17, 136-140 (2002).

[ 3] H. E. M. Dobson, E. M. Danielson, I. D. V. Wesep, Plant Spec. Biol., 14, 153-166 (1999).

[ 4] A. K. Hagan, M. E. Rivas-Davila, J. R. Akridge, J. W. Olive, J. Environ. Hort., 23 (2) 77-85 (2005).

[ 5] Y. Wensheng, S. Lihua, Z. Dezhi, G. Tieliu, T. Yuhui, Z. Weisen, J. Jilin For. Inst., 2, 7-11 (1992).

[ 6] L. Zhaolin, Z. Fanxzhi, C. Nengyu, X. Dunyuan, C. Yaozu, Chin. J. Chromatogr, 1 , 18-23 (1998).

[ 7] P. Yan-li, Z. Bing-zhen, M. Zong-hui, W. Yu-tuan, W. Ban-xiao, L. Qing,. L., Food and Drug 3 (2009).

[8] Y. Ueyama, S. Hashimoto, H. Nii, K. Furukawa, Fravour Fragr. J., 5 (2), 219-222 (1990).

[9] Y. Hashidoko, Phytochemistry, 43 (3), 535-549 (1996).

[10] M. Mahboubi, F. G. Bidgoli, Phytomedicine, 17(7), 548-550 (2010).

[11] A. Saad, M. Fadli, M. Bouaziz, A. Benharref, N.-E. Mezrioui, L. Hassani, Phytomedicine, 17(13), 1057-1060 (2010).

[12] S. Hemaiswarya, M. Doble, Phytomedicine, 16(11), 997-1005 (2009).

[13] N. Yayli, A. Yaşar, C. Güleç, A. Usta, S. Kolayl1, K. Coşkunçelebi, Ş. Karaoğlu, Phytochemistry, 66(14), 1741-1745 (2005).

[14] J. Yu, J. Lei, H. Yu, X. Cai, G. Zou, Phytochemistry, 65(7), 881-884 (2004).

[15] M. Skočibušić, N. Bezić, V. Dunkić, Food Chemistry, 96, 20-28 (2006).

[16]Y. Inoue, T. Hada, A. Shiraishi, K. Hirose, H. Hamashima, S. Kobayashi, Antimicrob Agents Ch, 49 (5), 1770-1774 (2005).

[17] M. Nagaki, T. Narita, H. Ichikawa, J. Kawakami, A. Nakane, Trans. Mater. Res. Soc. Jpn., 36 (1), 55-58 (2011).

[18] A. Nagayama, K. Yamaguchi, K. Watanabe, M. Tanaka, I. Kobayashi, and Z. Nagasawa, J. Infect. Chemother., 14, 383-392 (2008).

[19] S. Arakawa, T. Matsui, S. Kamidono, Y. Kawada, H. Kumon, K.Hirai, T. Hirose, T. Matsumoto, K. Yamaguchi, T. Yoshida, K. Watanabe, K. Ueno, A. Saito, and T. Teranishi, J Infect Chemother, 4, 97-106 (1998).

[20] A.Saito, J Infect Chemother, 1, 83-88
(1995).

[21]M. D’Arrigo, G. Ginestra, G. Mandalari, P. M. Furneri, G. Bisignano, Phytomedicine 17, 317-322 (2010).

[22]S. Hemaiswarya, M. Dole, Phytomedicine 16, 997-1005 (2009).

[23]M.-S. Pyun, S. Shin, Phytomedicine 13, 394-400 (2006).

[24]S. Hemaiswarya, A. K. Kruthiventi, M. Dole, Phytomedicine 15, 639-652 (2008).

[25]Q. L. Fivelman, I. S. Adagu, D. C. Warhurst, Antimicrob. Agents Chemother., $48,4097-4102$ (2004).

[26]R. J. Tallarida, J. Pharmacol. Exp. Ther. 298, 865-872 (2001).

(Received July 6, 2011; Accepted August 31, 2011) 